\title{
Positron Scattering on Atoms and Molecules in the Limit of Low Energy
}

\author{
G.P. Karwasz ${ }^{a, *}$, R.S. Brusa ${ }^{b}$, D. PliszkA ${ }^{c}$, Z. IdziaszeK $^{d}$ \\ AND H. NOWAKOWSKA ${ }^{e}$ \\ ${ }^{a}$ Institute of Physics, Nicolaus Copernicus University \\ Grudziądzka 5/7, 87-600 Toruń, Poland
}

${ }^{b}$ Physics Department, Trento University, Sommarive 14, 38050 Trento, Italy

${ }^{c}$ Institute of Physics, Pomeranian Pedagogical Academy, 76-200 Słupsk, Poland

${ }^{d}$ CNR-INFM BEC Center, 38050 Trento, Italy

and Center of Theoretical Physics, Polish Academy of Sciences

al. Lotników 32/46, 02-668 Warszawa, Poland

${ }^{e}$ Institute of Fluid Flow Machinery, Polish Academy of Sciences

Fishera 14, 80-952 Gdańsk, Poland

Starting from experimental cross-sections for positron scattering in argon and nitrogen, we examine different energy ranges. In the zero-energy limit the cross-section falls with energy and can be described by modified effective range theory for polarization potential. In a few $\mathrm{eV}$ range the cross-sections are constant vs. energy. As far as it is possible to force the elastic scattering phase shifts in a way that both experimental differential cross-sections are reproduced and the total cross-section remains constant in energy, such a model lacks the physical justification. Only the virtualpositronium model, developed recently by Gribakin, reproduces a constant dependence of the total cross-section in a few eV energy range.

PACS numbers: $34.85 .+\mathrm{x}, 39.90 .+\mathrm{d}$

\section{Introduction}

Positrons injected into solids thermalise in a short time $10^{-12}-10^{-11} \mathrm{~s}$ slowing down to a few tens of meV kinetic energy and then drift for relatively long time $\left(10^{-10}-10^{-7} \mathrm{~s}\right)$. Annihilation processes depend on the overlap of positron and electron wave functions. This overlap is determined by the integral crosssection for scattering, i.e. by some effective dimension of atoms, as seen by the colliding positron.

*corresponding author; e-mail: karwasz@science.unitn.it 
Quite recently it has been noticed [1] that in the limit of a few tens of $\mathrm{meV}$ the annihilation rates measured in gas phase are much higher than it would result from a number of electrons in the target. For example, in argon at $0.2 \mathrm{eV}$ the effective electronic number in atom is about 30, falling down to about 15 at $0.5 \mathrm{eV}$ and then remaining constant up to $2.5 \mathrm{eV}$ [1]. These annihilation rates are huge for bigger molecules like hydrocarbons, reaching for example the effective electronic number of 98000 for cyclohexane molecule [1]. One of the causes of such huge annihilation rates can be resonance processes, observed near thresholds for vibrational excitations [2], but the whole picture is still unclear. Here we analyze total cross-sections for positron scattering. These comprise also the cross-sections for annihilation, but up to the threshold for positronium formation $(8.9 \mathrm{eV}$ in $\mathrm{Ar}$, $8.7 \mathrm{eV}$ in $\mathrm{N}_{2}$ ) the elastic cross-section is the main part of the total one.

\section{Experimental}

Total cross-sections $\sigma$ have been measured by an absolute method (i.e. without arbitrary normalizations) in the range of $0.4-20 \mathrm{eV}$. The preliminary results for $\mathrm{Ar}, \mathrm{N}_{2}$, benzene, cyclohexane, and aniline have been presented in [3]. The cross-section is determined using de-Beer-Lambert attenuation law:

$$
I=I_{0} \exp (-p l \sigma / k T),
$$

where $I$ and $I_{0}$ is the current with and without gas in the scattering region, respectively, $l$ is the length of the scattering cell, $p$ is the gas pressure, $T$ is temperature of the gas, and $k$ is Boltzmann's constant.

Details of the experimental set-up have been given, for example, in Ref. [4]. Briefly, the spectrometer uses sodium ${ }^{22} \mathrm{Na}$ isotope as a positron source, a thin tungsten mono-crystal - as positron moderator, the electrostatic extraction optics in the region of moderator and a guiding magnetic field $(10 \mathrm{G})$ in the regions of the scattering cell (which is $10 \mathrm{~cm}$ long and has $1.5 \mathrm{~mm}$ entrance and exit apertures).

A rise of cross-sections in the zero-energy limit is controversial, in particular in the view of previous experiments and theories. In Fig. 1 we compare our crosssections in benzene with experiments by Sueoka [5-9] and collaborators, obtained by them in different time periods. Depending on experimental details, the total cross-sections by Sueoka show a rise [5] or a fall in the zero-energy limit [8]. We guess that these discrepancies depend on the magnitude of the guiding magnetic field used (varying between $3 \mathrm{G}$ and $27 \mathrm{G}[5]$ ). Our data show a rise, down to the lowest energy measured, in qualitative agreement with the ab-initio theory by Occhigrossi and Gianturco [10].

Our results in nitrogen, see Fig. 2 show a rise in the low energy limit, contrasting with other experimental data [11-14] and also with some theories [15-19]. The most probable reason for such underestimation of cross-sections in experiments is so-called angular resolution error. It is caused by the fact that due to the finite dimensions of the scattering cell exit, some of the scattered positrons can still reach the detector. This error is particularly big when guiding magnetic 


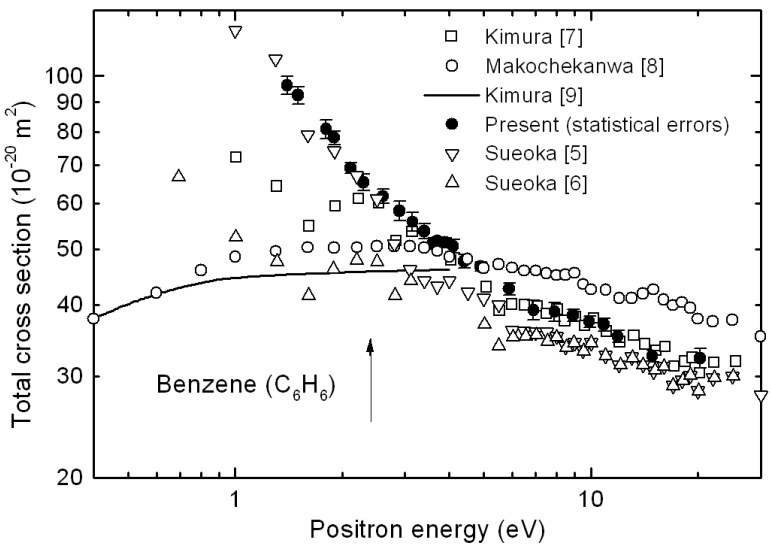

Fig. 1. Comparison of present cross-sections for positron scattering on benzene from Trento lab with other experiments [5-9] and theory [9].

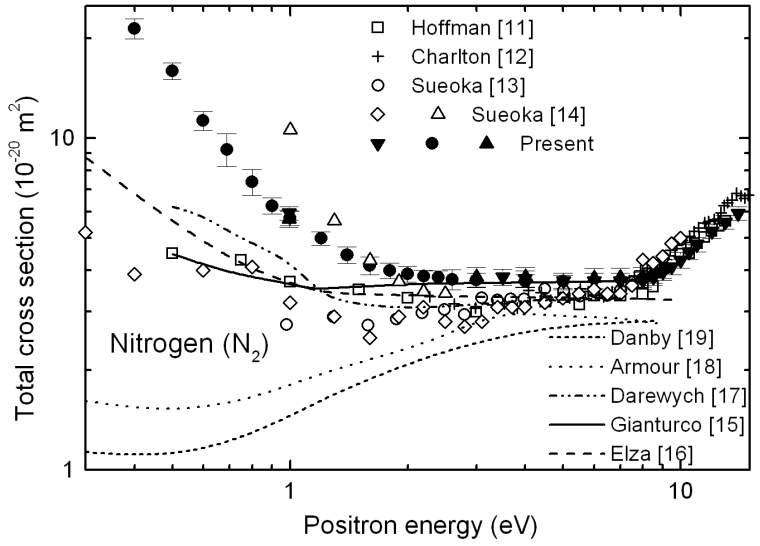

Fig. 2. Comparison of present cross-sections for positron scattering on nitrogen with other experiments [11-14] and theories [15-19]. For present data different experimental runs are shown by different symbols. For Sueoka data, triangles are his data corrected for angular resolution error, see Ref. [27] for details.

fields are used. However, to quantify this error, angular distributions of scattered positrons must be known. Nitrogen is one of a few fortuitous cases, where theoretical calculations extend down to zero energy and detailed angular distributions were given [20]. Using these data we can evaluate that with $8 \mathrm{~mm}$ diameter exit slits and $10 \mathrm{G}$ guiding field, the measured cross-sections at $1 \mathrm{eV}$ can be underestimated by a factor of three. In the case of the present apparatus, only at energies below $0.06 \mathrm{eV}$ the angular resolution error becomes significant (above 30\%). Therefore, from the experimental point of view, the rise of total cross-sections in the limit of zero energy seems to be proved, at least for targets like $\mathrm{N}_{2}, \mathrm{Ar}, \mathrm{H}_{2}$, benzene, aniline, cyclohexane, see experimental data for all these targets in [3, 4]. 


\section{Zero-energy range rise: analytical modified effective range theory}

The rise of the cross-sections would indicate importance of polarization potential in scattering. A theory of scattering on a short range ("effective range") potential combined with the polarization one was formulated already in the sixties of XX century [21]. It was the most frequently used in a form of expansion into the series of electron (positron) wave number $k$

$$
\sigma=4 \pi\left[A^{2}+\left(2 \pi / 3 a_{0}\right) \alpha A k+\left(8 / 3 a_{0}\right) \alpha A^{2} k^{2} \ln \left(k a_{0}\right)+B k^{2}+\ldots\right]
$$

with $a_{0}$ being Bohr's radius, $\alpha$ - dipole polarisability, $A$ being so-called scattering length and parameter $B$ depending on the effective range radius. (We recall that the wave number $k$ equals to positron momentum, if atomic units $a_{0}=h=m_{\mathrm{e}}=1$, with $m_{\mathrm{e}}$ being electron's mass, are used.) In spite of generally acceptance for the modified effective range theory, its practical applications for electron scattering gave rather poor results.

Idziaszek and Karwasz [22] have recently revisited the original formulation of scattering on the polarization potential through the Schrödinger equation in a spherical symmetry

$$
\left[\frac{\partial^{2}}{\partial r^{2}}+\frac{2}{r}+\frac{\partial}{\partial r}-\frac{l(l+1)}{r^{2}}+\frac{\left(R^{*}\right)^{2}}{r^{4}}+k^{2}\right] \Psi_{i}(r)=0,
$$

where $\Psi_{l}(r)$ denotes the radial wave function for the $l$-partial wave and $R^{*}=$ $e / \hbar \sqrt{\alpha \mu}$ describes a typical length related to the polarization interaction, $e$ is the electron charge, and $\mu$ is the reduced mass of positron-atom system.

In the paper [22] it was shown, how Eq. (3) can be reduced to Mathieu's modified differential equation (we recall Ref. [22] for a detailed description). By solving the set of Mathieu's equations for different partial waves, the phase shifts $\eta_{l}$ can be obtained analytically. We recall that the differential cross-section into the scattering angle $\theta$ is defined by the scattering amplitude $f(\theta)$ as $\mathrm{d} \sigma / \mathrm{d} \omega=|f(\theta)|^{2}$, where

$$
f(\theta)=\frac{1}{2 \mathrm{i} k} \sum_{l=0}^{\infty}(2 l+1)\left[\exp \left(2 \mathrm{i} \eta_{l}\right)-1\right] P_{l}(\cos \theta)
$$

with $P_{l}$ being Legendre polynomials.

The total cross-section is calculated as

$$
\sigma=\frac{4 \pi}{k^{2}} \sum_{l=0}^{\infty}(2 l+1) \sin ^{2} \eta_{l}
$$

The total cross-section obtained for argon is shown in Fig. 3 - one notes immediately that the present theory applies much better than the simplified series (2). The four parameters describing the effective range and the scattering length $A$ for the $s$ - and $p$-partial waves are given in Ref. [22]. In particular, the scattering length for the $s$-wave $\left(-5.58 a_{0}\right)$ agrees well with $a b$ initio calculation by McEachran et al. $\left(-5.30 a_{0}\right)$ [23]. The present analysis indicates quite high values of the cross-section in the limit of zero energy, about $110 \AA^{2}$ in $\mathrm{Ar}$ and as 


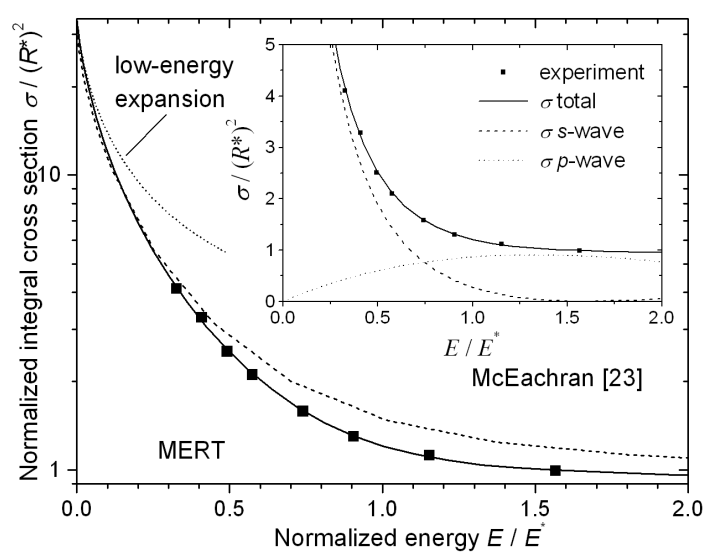

Fig. 3. A modified effective range theory with an analytic solution (MERT), as developed in Ref. [22], applied to Trento experimental data in $\operatorname{Ar}[4,27]$. The cross-section and collision energy are given in "characteristic" units, with $R^{*}=3.35 a_{0}\left(a_{0}-\right.$ Bohr units) and $E^{*}=1.211 \mathrm{eV}$ for argon. Short line is the simplified series, Eq. (2).

much as $290 \AA^{2}$ in $\mathrm{N}_{2}$. It shows also that $p$-wave scattering dominates over $s$-wave scattering already at about $1 \mathrm{eV}$, see Fig. 3 .

The advantage of this new formulation of the modified effective range theory is that it can be applied up to the energy of $1-2 \mathrm{eV}$, and therefore experimental cross-sections in this energy region can be used for the extrapolation towards zero energy. This analysis shows also that in the zero-energy limit these are polarization forces which dominate in the scattering process.

\section{A few eV energy range:}

\section{a hard-sphere model vs. virtual positronium formation}

Several experiments in argon in a few eV energy range differ somewhat in absolute amplitudes but all of them show constant, within experimental error bars, total cross-sections, see a detailed comparison in Ref. [4]. In spite of this, all theories show the total cross-section (i.e. integral elastic in this energy range) falling slightly with rising energy. A constant cross-section would be specific to hard-sphere scattering, and only in the classical approach. But in this case the angular distributions should be uniform, which contradicts existing measurements on targets like Ar and $\mathrm{N}_{2}$ [24].

To check whether the low-energy positron scattering can be described by a hard-sphere quantum mechanical model, we tried a following, somewhat textbook approach [25]. It is known that the integral cross-section for scattering on a hardsphere of radius $a$ changes from $4 \pi a^{2}$ in the zero-energy limit to $2 \pi a^{2}$ in the infinite energy limit, and the differential cross-sections show interference patterns. The aim of the present check was to establish to what extent the integral cross-section 
changes in the range of a few $\mathrm{eV}$ and whether differential cross-sections show interference patterns.

In the quantum model of scattering on a hard sphere, the $s$-phase shifts can be obtained from the expression $\eta_{0}=-k a$ and higher phase shifts can be calculated from the formula

$$
\tan \eta_{l}=(-1)^{l-1} \frac{J_{l+1 / 2}(k a)}{J_{-l-1 / 2}(k a)},
$$

where $J$ are Bessel functions. For the present check we tried "reasonable" values of hard-sphere radius $a$, i.e. in the range of a few angstroms. Some of the trials are shown in Fig. 4, at a given energy of $6.75 \mathrm{eV}$ (corresponding to $k=1.33 \AA^{-1}$ ) and for selected values of the $k a$ product. It is clear from Fig. 4 that in order to reproduce a pronounced interference pattern like that observed for $\mathrm{N}_{2}$ at $6.75 \mathrm{eV}$, the product $k a$ should be higher than 5.0. This, in turn, would correspond to hard-sphere radii much exceeding the values of "atomic dimensions" as obtained from viscosity measurements, van der Waals radii $\left(1.47 \AA\right.$ for $\left.\mathrm{N}_{2}\right)$ and so on (see also discussion in Ref. [26]). It would also yield the integral cross-section exceeding by a factor of ten or more the values measured $[4,27]$ in a few eV energy range. Clearly, the quantum mechanical hard-sphere model is not applicable to low-energy positron scattering.

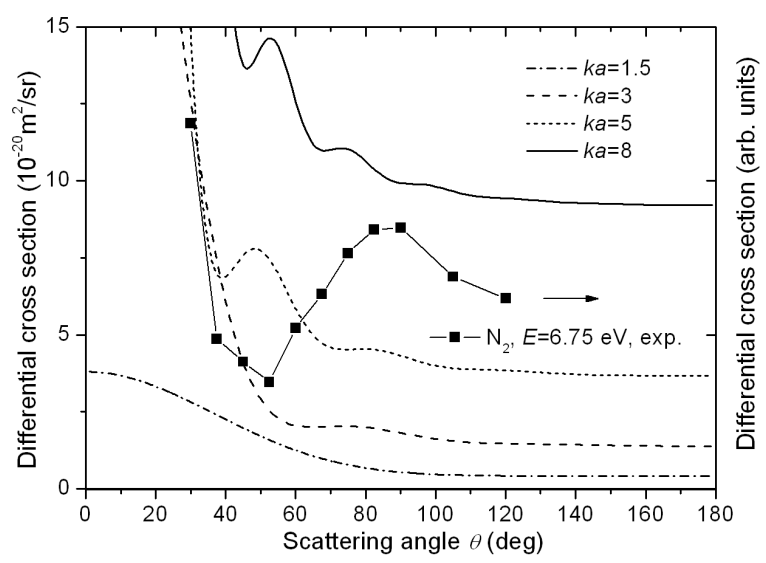

Fig. 4. Differential cross-sections (Eq. (4)) using phase shifts from hard-sphere quantum model, Eq. (6) for several values of $k a$ ( $a$ being the radius of the sphere) compared to the experimental (relative) differential cross-sections for positron scattering on $\mathrm{N}_{2}$ [24]. Calculations have been done for the collision energy of $6.75 \mathrm{eV}$ (corresponding to the wave number $k=1.33 \AA^{-1}$ ). The given range of the $k a$ product corresponds to the hard-sphere radii $a$ between 1.1 and $6.0 \AA$. Only high values of $a$ give an interference pattern in the differential cross-section, but then the integral cross-section (Eq. (5)) exceeds experimental values (Ref. [27]) by a few folds. The left ordinate scale corresponds to theoretical values, the right one (in relative units) to the experimental values. 
Therefore we checked an alternative, phenonomenological approach. We did not calculate phase shifts from a given potential but adjusted them in an arbitrary way with two constraints. The first one was to maintain the integral cross-section constant in the 5-15 eV range, the second one was to reproduce the shape of experimental differential cross-sections [24] (which, we recall, were obtained in relative units).

Presently fitted differential cross-sections at $5 \mathrm{eV}$ and $15 \mathrm{eV}$ are compared to experimental data [24] and ab-initio theory [23] in Fig. 5. We have adjusted only four partial waves phase shifts but the present trial reproduces differential crosssections in the measured angular range not worse than the ab-initio calculations by McEachran et al. [23]. The integral cross-section in this model amounts to $3.0 \AA^{2}$ between 5 and $15 \mathrm{eV}$, in agreement with our experiment [27]. The phase shifts calculated presently, apart from those for $p$ and $d$ waves at $15 \mathrm{eV}$, agree also pretty well with those of McEachran et al. [23], see Table.

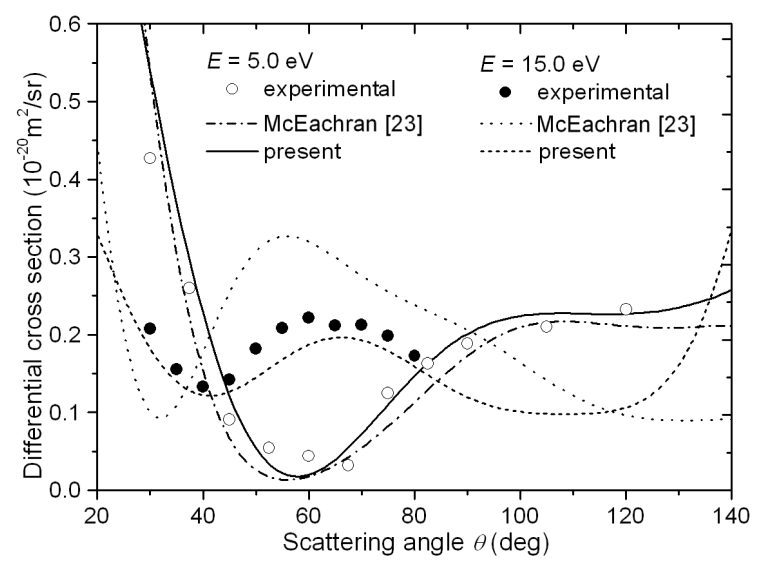

Fig. 5. Phenomenological approximation of the experimental differential cross-sections for positron-Ar scattering [24] at two chosen energies: $5 \mathrm{eV}$ and $15 \mathrm{eV}$. We omit a figure for $8.7 \mathrm{eV}$, with similarly good approximation of experimental data [24]. The constraint for this approximation was a constant integral cross-section, of $3.0 \AA^{2}$, equal to the value measured between about 2 and $8 \mathrm{eV}$ in Ref. [27]. Experimental (relative) values were normalized by arbitrary factors, to allow a comparison with present theoretical curves.

The only problem with this approach is that no physical potential has been attributed to the phase shifts. However, this result indicates that taking into account the elastic scattering solely, one could reproduce the constant integral cross-section up to the positronium formation threshold.

At this point we quote the only other model reproducing constant crosssections, namely the recent many-body theory approach developed by Ludlow and Gribakin [28] who introduced explicitly the process of virtual positronium formation. Briefly, an incoming positron is closely coupled to one of the target 
TABLE

Phase shifts (in rad) for positron-argon scattering, used for differential (Eq. (4)) and total cross-sections (Eq. (5)) at three collision energies. Upper rows for each energy are from the present phenomenological model, in which phase shifts were adjusted to reproduce experimental (relative) differential cross-sections [23] and a constant (absolute) integral cross-sections $\left(3.0 \AA^{2}\right)$, as it was measured in Trento experiment [27]. Values in lower rows for each energy are from $a b$ initio calculations [23].

\begin{tabular}{c|c|c|c|c|c}
\hline \hline $\begin{array}{c}\text { Collision energy } \\
(\mathrm{eV})\end{array}$ & \multicolumn{5}{|c}{ Phase shift $\eta_{l}$} \\
\cline { 2 - 6 } 5.0 & -0.2548 & 0.2478 & 0.1082 & 0.0401 & \\
& -0.2198 & 0.2339 & 0.1231 & 0.0449 & 0.0194 \\
\hline 8.7 & -0.6161 & 0.2094 & 0.0454 & 0.1012 & \\
& -0.4863 & 0.1520 & 0.1705 & 0.0801 & 0.0364 \\
\hline \multirow{2}{*}{15.0} & -0.8552 & 0.23038 & -0.0384 & 0.1728 & \\
& -0.7812 & -0.0094 & 0.1724 & 0.1209 & 0.0638
\end{tabular}

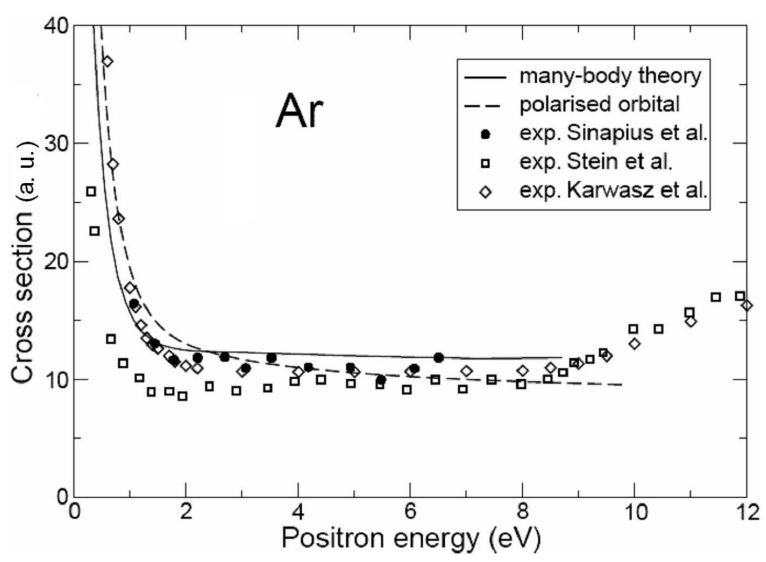

Fig. 6. Integral cross-section (in atomic units) for positron scattering on argon in the virtual positronium model ("many-body theory", Ref. [29]) compared to the polarizedorbital theory, Ref. [23] and experimental values (see Ref. [27] for labels). This picture is courtesy of Prof. Gleb Gribakin.

electrons and this modifies the whole distribution of electrons in the target. The application of a series of perturbative corrections to the electronic density of atom, corresponding to the explicit inclusion of polarization and/or correlation effects reproduces our experimental constant total cross-section, see Fig. 6 (this figure was kindly allowed to us by Ludlow and Gribakin [29]). We are not aware of other similar calculations. 


\section{Conclusions}

Combining the analysis of experimental results with a re-visited theoretical model allows us to obtain a congruent picture of positron scattering in the low energy range. The recent measurements indicate that cross-sections for positron scattering rise in the limit of zero energy. An analytical solution of the effective range problem with the polarization potential included [22] allows us to extrapolate experimental cross-sections in the $0.5-2 \mathrm{eV}$ energy range towards zero energy. The values of zero-energy cross-sections obtained in this way exceed $100 \AA^{2}$ for $\mathrm{Ar}$ and $200 \AA^{2}$ in $\mathrm{N}_{2}$.

At higher energies, in order to reproduce constant cross-sections up to the free-positronium threshold, the formation of virtual positronium must be allowed for. Such pioneer calculations have been recently developed by Ludlow and Gribakin [29]. Finally we note that in the higher energy range, i.e. above the positronium formation threshold, adding the experimental cross-sections for positronium formation in $\mathrm{Ar}$ [30] to a constant-value elastic cross-section, as deduced from the above phenomenological or "virtual positronium" models, allows us to reproduce experimental total cross-sections from recent Trento measurements [27] within the error bars.

\section{Acknowledgment}

We acknowledge the kind permission of prof. Gleb Gribakin to present his unpublished data in Fig. 6.

\section{References}

[1] T.J. Murphy, C.M. Surko, Phys. Rev. Lett. 67, 2954 (1991).

[2] J.P. Sullivan, S.J. Gilbert, J.P. Marler, L.D. Barnes, S.J. Buckman, C.M. Surko, Nucl. Instrum. Methods B 192, 3 (2002).

[3] G.P. Karwasz, D. Pliszka, R.S. Brusa, C. Perazzolli, Acta Phys. Pol. A 107, 666 (2005).

[4] G.P. Karwasz, D. Pliszka, A. Zecca, R.S. Brusa, Nucl. Instrum. Methods Phys. Res. B 240, 666 (2005).

[5] O. Sueoka, J. Phys. B, At. Mol. Phys. 21, L631 (1988).

[6] O. Sueoka, M.K. Kawada, M. Kimura, Nucl. Instrum. Methods Phys. Res. B 171, 96 (2000).

[7] M. Kimura, O. Sueoka, A. Hamada, Y. Itikawa, Adv. Chem. Phys. 111, 537 (2000).

[8] C. Makochekanwa, O. Sueoka, M. Kimura, Phys. Rev. A 68, 32707 (2003).

[9] M. Kimura, C. Makochekanwa, O. Sueoka, J. Phys. B, At. Mol. Opt. Phys. 37, 1461 (2004).

[10] A. Occhigrossi, F.A. Gianturco J. Phys. B, At. Mol. Opt. Phys. 36, 1383 (2003).

[11] K.R. Hoffman, M.S. Dababneh, Y.-F. Hsieh, W.E. Kauppila, V. Pol, J. Smart, T.S. Stein, Phys. Rev. A 25, 1393 (1982). 
[12] M. Charlton, T.C. Griffith, G.R. Heyland, G.L. Wright, J. Phys. B, At. Mol. Opt. Phys. 16, 323 (1983).

[13] O. Sueoka, S. Mori, J. Phys. Soc. Jpn. 53, 2491 (1984).

[14] O. Suoeka, S. Hamada, J. Phys. Soc. Jpn. 62, 2669 (1993).

[15] F.A. Gianturco, P. Paioletti, J.A. Rodriguez-Ruiz, Z. Phys. D 36, 51 (1996).

[16] B.K. Elza, T.L. Gibson, M.A. Morrison, B.C. Saha, J. Phys. B, At. Mol. Opt. Phys. 22, 113 (1989).

[17] J.W. Darewych, J. Phys. B, At. Mol. Opt. Phys. 15, L415 (1982).

[18] E.A.G. Armour, M. Plummer, J. Phys. B, At. Mol. Opt. Phys. 24, 4463 (1991).

[19] G. Danby, J. Tennyson, J. Phys. B, At. Mol. Opt. Phys. 24, 3517 (1991).

[20] C.R.C. de Carvalho, M.T. do N. Varella, M.A.P. Lima, E.P. da Silva, J.S.E. Germano, Nucl. Instrum. Methods Phys. Res. B 171, 33 (2000).

[21] T.F. O'Malley, Phys. Rev. A 130, 1020 (1963).

[22] Z. Idziaszek, G.P. Karwasz, Phys. Rev. A 73, 064701 (2006).

[23] R.P. McEachran, A.G. Ryman, A.D. Stauffer, J. Phys. B 12, 1031 (1979).

[24] W.E. Kauppila, T.S. Stein, Adv. At. Mol. Opt. Phys. 26, 1 (1990).

[25] N.F. Mott, H.S.W. Massey, The Theory of Atomic Collisions, Clarendon Press, Oxford 1965.

[26] G.P. Karwasz, Eur. Phys. J. D 35, 267 (2005).

[27] G.P. Karwasz, D. Pliszka, R.S. Brusa, Nucl. Instrum. Methods Phys. Res. B 247, 68 (2006).

[28] G.F. Gribakin, J. Ludlow, Phys. Rev. A 70, 032720 (2004).

[29] J. Ludlow, G.F. Gribakin, private communication.

[30] J.P. Marler, J.P. Sullivan, C.M. Surko, Phys. Rev. A 71, 022701 (2005). 